\title{
PHILOSOPHY OF CLASSIFICATION FOR WN STARS
}

\author{
LINDSEY F. SMITH ${ }^{1}$, MICHAEL M. SHARA ${ }^{2}$ and \\ ANTHONY F.J. MOFFAT ${ }^{3}$ \\ ${ }^{1}$ School of Physics, University of Sydney, Australia \\ ${ }^{2}$ Space Telescope Science Institute, Baltimore, MD, U.S.A. \\ ${ }^{3}$ Department de Physique, Université de Montreal, Canada
}

\begin{abstract}
The unsatisfactory present state of the classification system for WN stars is summarised and the three dimensions of a new system are presented and justified.
\end{abstract}

Key words: stars: Wolf-Rayet - classification

The classification of WN stars created by Beals (1938) and modified by Hiltner \& Schild (1966) and Smith (1968) has not proven satisfactory, resulting in a high proportion of stars which do not fit and an overall impression of one star $=$ one type (e.g., Conti et al. 1983). The purpose of classification is to separate stars into groups whose spectra and, by implication, physical parameters are nearly identical. The failure to arrive at such groupings for the WN stars lies with the specification of the current classification system. A revised system, detailed in a forthcoming paper, successfully sorts nearly all spectra into uniform groups. Experience with classification of lower mass stars showed that a star's mass and composition - and hence spectrum - are related in a nearly singled-valued way to initial mass and age. The two dimensional classification of ordinary $\mathrm{B}$ to $\mathrm{M}$ stars relates directly to temperature and luminosity. The WC stars, although their history is varied, also arrive at a stage defined simply by present mass and composition. The known diversity of WC spectra is adequately described by a one parameter classification system (Smith et al. 1990), although it is expected that two dimensions will eventually be necessary and desirable.

We now know that WN stars represent an evolutionary stage, intermediate between massive $O$ stars and WC stars. The $O$ stars evolve rapidly by mass loss, eventually revealing material with a high proportion of helium the WN stage. Further mass loss removes the remaining material outside the pure helium (and higher elements) core at which point the star displays a WC spectrum. Since the rate of mass loss depends on the mass, metallicity and binary/single state of the initial star, the mass and surface composition may be a complex function of previous evolution. It is not surprising that we should need two or, as suggested here, three dimensions to classify the spectra into uniform groups.

Beals' (1938) and Smith's (1968) systems for both WN and WC sequences were one dimensional - seeking a definition by ionization only. Hiltner \& Schild (1966) split the WN stars into two sequences, the narrow-weak line 
(A) and broad-strong line (B) stars. The physical validity of separation by line strength was recently validated (Hamann et al. 1993). The probable importance to the subtypes of $\mathrm{H} / \mathrm{He}$ ratio was recognized by Smith (1973) and it significance in terms of physical parameters validated by Hamann et al. (1993). We propose presence of hydrogen as the third dimension of the classification. Redefinition of the classification is timely because of the advances in our understanding outlined above and also because of the advent of linear detectors with wide wavelength sensitivity. It is now easy to measure line ratios quantitatively and for lines which are well separated in wavelength.

Specifically, the three dimensions are: (1) ionization - defined, for the WN3-8, stars primarily by the ratio of He I $5875 \AA$ to He II $5411 \AA$. As the primary criterion, this eliminates confusion. Usual nitrogen ratios are also defined, little changed from previous systems; these are useful as conspicuous guides and are important, in practice, at the ends of the sequence when one or other of the He lines becomes weak; (2) line-width - with the boundary at FWHM $4686 \AA=30 \AA$. Width is a more useful criterion than strength (used by Hamann et al. 1993) since it is unaffected by blending of the WR spectrum with another; the two are closely related - a matter explored more in the forthcoming paper on this subject; and (3) presence of $\mathrm{H}$ as judged by the bumpiness of the Pickering decrement - specifically: the $\mathrm{He}+\mathrm{H} 4861 \AA$ and $4340 \AA$ lines greater than the geometric mean of the pure He lines on either side. Observational uncertainty, judged by differences between various spectra that we have inspected, indicate (in agreement with Hamann et al. 1993) that the reliable detection limit by this method is about $\mathrm{H} / \mathrm{He}=0.5$ by number. Fortunately, this appears also to be the dividing line where the $\mathrm{H}$ presence has a significant effect on derived physical parameters.

For classes later than 8 , classification by helium emission lines fails. It could be well argued that a WR spectrum is one with He II $5411 \AA$ strongly in emission - apparently a good indicator of high helium, low hydrogen abundance. The WN9-11 stars appear to have hydrogen abundance intermediate between that of $\mathrm{O}$ stars and WR stars (Crowther et al. these proceedings) and could be classified as either. However, the classification of WN9-11 by Crowther et al. (1994) appears well defined and connected to the core WR classes - both in terms of spectral appearance and evolutionary stage.

\section{References}

Beals, C.S. 1938, Trans. IAU 6, 248

Crowther, P.A., Hillier, D.J., Smith, L.J. 1994, $A \& A$ in press

Conti,P.S., Leep, E.M., Perry, D.N. 1983, $A p J$ 268, 228

Hamann, W.-R., Koesterke, L., Wessolowski, U. 1993, $A \& A$ 274, 397

Hiltner, W.A., Schild, R.E. 1966, ApJ 143, 770

Smith, L.F. 1968, MNRAS 138, 109

Smith, L.F. 1973, in: M.H.V. Bappu \& J. Sahade (eds.), Wolf-Rayet and High Temperature

Stars, Proc. IAU Symp. No. 49 (Dordrecht: Reidel), p. 15

Smith, L.F., Shara, M.M., Moffat, A.F.J. 1990, $A p J$ 348, 471 